\title{
Enseigner la sociologie du travail artistique par l'ethnographie
}

Retour sur une expérience pédagogique à l'Université de Lausanne

Teaching the sociology of artistic work through ethnography. An educational experience at the University of Lausanne reconsidered

Enseñar la sociología del trabajo artístico a través de la etnografía. Repasando una experiencia pedagógica en la Universidad de Lausanne

Pierre-Emmanuel Sorignet et Marc Perrenoud

\section{(2) OpenEdition}

\section{Journals}

Édition électronique

URL : http://journals.openedition.org/bssg/257

DOI : $10.4000 /$ bssg. 257

ISSN : $2490-9424$

Éditeur

Presses universitaires de Vincennes

Référence électronique

Pierre-Emmanuel Sorignet et Marc Perrenoud, « Enseigner la sociologie du travail artistique par

l'ethnographie », Biens Symboliques / Symbolic Goods [En ligne], 2 | 2018, mis en ligne le 12 avril 2018, consulté le 04 mars 2021. URL : http://journals.openedition.org/bssg/257 ; DOI : https://doi.org/ $10.4000 /$ bssg. 257 


\title{
Enseigner la sociologie du travail artistique par l'ethnographie Retour sur une expérience pédagogique à l'Université de Lausanne
}

\author{
Pierre-Emmanuel Sorignet | Marc Perrenoud
}

En juin 2015 notre université nous a proposé de prendre en charge un " atelier de recherche » en master consacré à la sociologie du travail artistique entre février et juin 2016. Ce nouveau support comprenant 112 heures d'enseignement en présentiel et comptant pour 12 crédits ECTS, il nous est rapidement apparu que nous avions là l'occasion d'élaborer un « scénario pédagogique » original en montant un spectacle avec nos étudiant·e.s. Considérant qu'une part importante de ces dernier.e.s, inscrit.e.s dans l'orientation "Culture et communication », avaient de bonnes chances d'intégrer les métiers de l'intermédiation culturelle et sachant que les professionnel.le.s de la culture entretiennent souvent un rapport " fantasmé » à l'activité artistique (Dubois 2013), il nous semblait intéressant de leur proposer de se former à la sociologie du travail artistique à travers une pratique réflexive du travail artistique.
Respectivement danseur-chorégraphe (Sorignet) et musicien (Perrenoud) professionnels, spécialistes de l'ethnographie de ces métiers et toujours actifs dans ces espaces, nous avons pris contact avec des responsables de structures de répétition et de diffusion à Lausanne ainsi qu'avec des danseurs, chorégraphes ou musiciens dans nos réseaux professionnels pour établir un programme de travail relativement simple : tous les mardis du semestre, de 9 heures à 18 heures, nous avons travaillé dans une salle de danse équipée d'une sono, alternant travail physique, échauffements et exercices propres à la danse contemporaine, moments de cours « classiques " de sociologie, tables rondes thématiques autour des différents corps de métiers du spectacle vivant (administration, programmation, technique, mise en scène), dialogue avec les artistes invité e.s sur leur parcours et travail sous leur direction. À la fin du semestre, nos vingt étudiant·e.s ont présenté un spectacle deux soirs d'affilée dans un théâtre lausannois devant 150 personnes à chaque fois. 
Ce court texte vise à opérer un premier retour sur l'expérience pédagogique que nous avons menée en commençant par voir comment les étudiant.e.s de master de sociologie ont reçu le projet tout au long du semestre. Nous avons, en effet, pu en reparler avec certain'e.s participant $\cdot e \cdot s$ au cours des mois qui ont suivi, ce qui nous a permis d'interroger notamment les conditions de transmission de l'approche ethnographique par rapport à ce qu'avait pu être leur formation aux sciences sociales jusqu'alors («1. Ce que l'art fait à la sociologie »). Ensuite nous exposerons brièvement comment notre démarche s'est insérée dans le jeu de la scène lausannoise du spectacle vivant et de la danse contemporaine en particulier («2. Ce que la sociologie fait à l'art»), avant de conclure en envisageant les perspectives pour les prochaines occurrences de cet enseignement biennal.

\section{Ce que l'art fait à la sociologie : sens pratique et mise en jeu de soi}

Prendre le prétexte d'un projet artistique pour transmettre le regard sociologique et la méthode ethnographique était un projet ambitieux. Nous montrerons ici comment a opéré tout le travail d'incorporation réflexive qui était central pour nous, ainsi que les résistances auxquelles nous avons pu nous heurter ${ }^{1}$.

1 Précisons que parallèlement au travail sur le plateau, nous souhaitions sensibiliser les étudiant.e.s à d'autres dimensions du travail dans les mondes de l'art (Becker 1988 [1982]) en leur faisant mener une enquête par observations et entretiens sur l'un des différents métiers du théâtre qui accueillait cette expérience pédagogique (administration, technique communication, cuisine, etc.). Cet aspect du travail, plus classique, n'est pas abordé dans le présent article.

\section{Incorporer l'expérience artistique}

Durkheim, dans ses premiers écrits sur la pédagogie, se défiait des arts comme vecteur de transmission en soulignant que « l'art se détourne de la vie morale » car il est éloigné du réel, fondement même de son objectif pédagogique qui est de former des citoyens à faire société (Durkheim 2005 [1922] : 2012). Néanmoins, persuadés comme Durkheim que le savoir sociologique permet à l'enseignant·e d'être davantage conscient.e des enjeux sociaux propres à toute mission éducative, il nous semblait, au contraire, que proposer un projet artistique commun à nos étudiant·e.s permettrait, au-delà du contenu proprement sociologique de l'approche, d'atteindre l'« éducation sociale » qui est au cœur du projet durkheimien : " II faut apprendre aux jeunes qu'il est, audelà des fins "personnelles", des fins "impersonnelles", "supraindividuelles", d'un intérêt collectif » (Fillioux 1994).

En ce sens, l'expérience pédagogique portée par le projet chorégraphique a structuré un « nous " collectif tout au long des séquences de répétitions passées à " souffrir et rire ensemble " (une étudiante fortement investie dans le projet), jusqu'aux représentations, point d'orgue du sentiment d'appartenance au groupe. Comme le souligne l'une des participantes qui revient un an et demi plus tard sur sa perception de l'atelier: " c'était une expérience de vie, bien au-delà de l'expérience scolaire ». L'entraide mutuelle, la coexistence, proche de la promiscuité corporelle dans les loges, dans le rapport quotidien à l'autre (embrassades, toucher), prolongent l'expérience du plateau et de la routine du travail dans la danse. L'éclosion d'une identité collective à travers un sentiment d'appartenance (Avanza \& Laferté 2005) dont la matrice est le projet artistique apparaît de manière récurrente dans les extraits de journaux de terrain rendus aux enseignants, comme dans les discussions informelles avec les étudiant·e.s. En cela l'ambition 
de dépasser le cadre scolastique d'un enseignement universitaire est atteinte au sens où Durkheim voyait dans « la fonction propre de l'éducation ", celle " de cultiver l'homme, de développer les germes d'humanité qui sont en nous » (Durkheim 1969 [1938]).

L'usage d'une pédagogie articulant enseignement des principaux travaux et concepts de la sociologie de l'art et des professions artistiques avec une immersion dans ce que nous voulions donner à voir comme l'ordinaire du travail dans le spectacle avait un double objectif. II s'agissait d'une part de confronter les étudiant·e.s à la pratique de l'ethnographie et plus spécifiquement de l'observation participante qui est l'un des ressorts de notre approche des professions artistiques. D'autre part, nous voulions les « sensibiliser par corps » à une sociologie des dispositions, méconnue par la plupart compte tenu de l'importance dans les maquettes de ce master d'une sociologie pragmatique adossée à un programme méthodologique et théorique proche de l'ethnométhodologie. Notre pari était qu'en éprouvant « par corps " l'activité des danseurs et danseuses, les étudiant·e.s pourraient à la fois vivre la façon dont se déploie un « habitus professionnel » de la danse, un ensemble de façons de faire, dire et penser propres à ce métier, et l'objectiver dans le même temps à travers la tenue régulière du journal de terrain et les échanges avec les enseignants sur ce qui se déroulait tout au long des séances spécifiquement consacrées à l'apprentissage corporel. L'idée que personne n'est danseur de naissance et que cela procède d'une socialisation intense, d'une transformation de soi de type vocationnelle (Suaud 1978, Sapiro 2007, Laillier 2011) par le biais d'institutions de formation, était directement mise en application dans l'observation sur soi-même de l'acquisition progressive et parfois spectaculaire de techniques du corps qui leur paraissaient souvent au départ hors de portée. Se rouler par terre, chuter, occuper l'espace du plateau et avoir conscience de celui-ci, apprendre à accepter le toucher, le contact, puis le poids de l'autre, tout cela faisait partie d'une fabrique sociale en train d'opérer et qui a influé sur le style de vie de la grande majorité des étudiant·es au fur et à mesure que le semestre avançait (façons de se tenir, de s'habiller, de parler, pratiques culturelles, etc.). Comme le souligne l'une d'elles : « on était pris par ça, on vivait dans notre quotidien le projet. On en parlait tout le temps, c'était le sujet central de nos conversations ". Certain.e.s même prolongeaient les ateliers du mardi en répétant entre eux des mouvements qu'ils/elles voulaient pleinement assimiler. La prise de conscience du fait que devenir danseur ou danseuse résulte de dispositions acquises par le travail était l'un des premiers objectifs de notre enseignement, de même que l'idée que cette acquisition de techniques du corps peut se prolonger - hors de toute compréhension mentale (Wacquant 2005) - dans une façon de se mouvoir au quotidien, de porter son corps dans l'espace social. Certain.e.s étudiant·e.s le retraduisaient alors dans l'objectivation spontanée de réactions littéralement extraordinaires. Ainsi Violette, 23 ans, relate un soir la tentative de vol de son sac dans un fast-food par deux hommes : « j'ai senti, parce que j'étais alerte dans mon corps, qu'on essayait de me voler mon sac... Mon corps s'est mis à courir tout seul et j'ai senti que j'étais entraînée et rapide. Moi qui suis peureuse, j'avais confiance dans mon corps et ça c'était tous les entraînements qu'on faisait. Ils ont laissé tomber. " Magali, 22 ans, raconte qu'ils et elles sont quelques-un'e.s à reproduire dans l'espace public des séquences de répétitions, allant jusqu'à se filmer et mettant ainsi en exergue leurs nouvelles capacités motrices mais aussi un sens de l'exposition publique qui ne leur était jusqu'alors pas familier : « parfois, on se mettait à chuter, tourner sur nous-mêmes dans le hall du métro, sans gêne, c'était super ! »

En outre, la réception différenciée, telle que retranscrite dans les journaux de terrain et les interactions à chaque séance, à la fois de l'expérience corporelle et de la mise en scène de soi dans le 
cadre du projet de représentation finale permet de saisir que les dispositions à acquérir ces nouvelles dispositions scéniques ne sont pas les mêmes selon les trajectoires sportive et scolaire suivies par les étudiant·e.s et, plus généralement, selon les ressources sociales dont elles/ils disposent. Entre Louis ( 23 ans, issu de la classe moyenne supérieure vaudoise, très sportif), Daria (25 ans, issue de la bourgeoisie russe, formée à la danse classique) et Agnès (38 ans, mère de famille, corpulente, en reprise d'étude), par exemple, la relative objectivation des dispositions de chacun'e a permis de dépasser l'inégale distribution des ressources (capital corporel en particulier) pour produire différentes formes de présence scénique tout aussi « intéressantes », «pertinentes » ou «importantes ».

Ainsi le processus collectif d'acculturation au travail chorégraphique et scénique a opéré tout au long du semestre. Un des aspects les plus saisissants concerne le rapport à la pudeur dont on sait depuis les travaux de Norbert Elias qu'il est l'un des régulateurs des rapports sociaux (Elias 1973 [1939]), en particulier des rapports sociaux de sexe, dans la mesure où la pudeur est fondée sur la maîtrise des pulsions et de la violence potentielle auxquelles cellesci peuvent conduire. Au début du semestre, filles et garçons se changeaient dans des espaces bien séparés, opposant un obstacle visuel au corps de l'autre. Au fur et à mesure des séances, cette gêne a progressivement et collectivement disparu. Au moment des représentations, les loges du théâtre mélangeaient dans un joyeux brouhaha filles et garçons unis par l'effervescence collective liée à la représentation, et dont les corps plus ou moins dénudés étaient devenus un outil de travail.

Il s'agissait bien, en reprenant le programme de Loïc Wacquant à la suite de Pierre Bourdieu, de faire du concept d'incorporation un dispositif à la fois méthodologique et pédagogique dans le cadre de cet atelier articulant expérience par corps et transmission de ce qui fait le cœur de l'objectivation participante, la réflexivité.

\section{Transmettre : l'ethnographie par corps}

En effet, il nous semble que la réflexion sur les pratiques d'observation et, à leur suite, d'écriture participe de la question de la transmission auprès d'étudiant·e.s accculturé.e.s non seulement au savoir scolaire, mais surtout à ses modalités d'acquisition dans le cadre de cours relativement standardisés. Déplacer le lieu de la transmission du savoir et surtout en transformer le cadre cognitif est apparu au fil de ce semestre comme un enjeu éducatif et épistémologique essentiel. En effet, faire de la connaissance sociologique un savoir empirique dont l'armature théorique ne se saisit pas par le seul jeu scolastique de la dialectique conceptuelle (avec ses figures imposées en matière de sociologie de la culture: "Bourdieu vs. Hennion ou Menger ", "Becker vs. Elias », etc.) supposait que le travail même de l'ethnographe devait être expérimenté, en situation et collectivement par les étudiant·e·s. Ce déplacement spatial et cognitif permettait aussi de leur suggérer que le savoir scolaire n'était pas le savoir scientifique mais « une version passée au rouleau compresseur, faite de phrases disjointes et dont toute théorie a été exprimée dans le processus d'aplatissement " (Delbos \& Jorion 1984). Dans la réflexion sur les rapports entre théorie et ethnographie, la question de la transmission du travail de recherche auprès d'étudiant·e.s en sciences sociales n'est, effectivement, que peu abordée. Ainsi, expliciter les ressorts du travail ethnographique et les conditions institutionnelles dans lesquelles il est enseigné apparaissait comme une façon de prolonger la réflexion sur les pratiques d'écritures en ethnographie en l'intégrant à la sphère pédagogique. 
Réfléchir aux conditions de l'enseignement de la pratique ethnographique, c'est aussi aborder la place de son corps dans le rapport au terrain d'observation. Plus précisément, il faut pouvoir identifier pour chaque étudiant.e une histoire scolaire et familiale qui prédispose plus ou moins à accepter des formats pédagogiques mettant à distance les prérequis scolaires habituellement mobilisés et soulignant les politiques du corps engagées par les institutions scolaires (Faure 2000, Faure \& Garcia 2005). Les dispositions scolaires sont ainsi indissociablement cognitives et rattachées aux processus d'incorporation de la norme scolaire et font de l'école une structure " d'intégration logique » (Bourdieu 1967) qui sert de principe de sélection et d'appréciation des formes légitimes ou illégitimes de diffusion des savoirs. Ainsi avonsnous fait de la sociologie en $t$-shirt et survêtement, discuté des logiques dispositionnelles et interactionnelles en jeu dans des exercices physiques tout en les réalisant ; ainsi nous sommesnous assis en tailleur pendant quelques minutes pour tel ou tel point théorique en lien avec ce que nous venions de traverser au cours d'un entraînement (sur les notions de tenue, de déférence ou de gêne chez Goffmann, ou bien sur les dynamiques esthétiques propres au champ de la création contemporaine, ou encore sur les rapports employeur.e/employé·e.s qui peuvent caractériser les relations entre un'e metteur'e en scène et ses interprètes). Nous avons, en somme, développé tout au long du semestre des pratiques contrevenant aux usages de la pédagogie universitaire classique et nous avons parfois eu des difficultés sinon à les faire accepter par les étudiant $\cdot e \cdot s$, du moins à leur faire entendre que ce que nous voulions leur transmettre, de cette manière informelle et inhabituelle, devait cependant être considéré comme un savoir légitime à s'approprier en tant que tel. Cette difficulté s'est particulièrement vérifiée dans un malentendu qui a marqué nombre d'étudiant·e.s dans cet atelier si particulier.

\section{Échec relatif de la transmission d'une sociologie des dispositions et du champ}

La prise de conscience réflexive du processus d'incorporation n'a pas été effective pour tou·te·s nos étudiant·e.s. On peut proposer deux hypothèses : tout d'abord comme le souligne Pierre Bourdieu " toute perception artistique implique une opération consciente ou inconsciente de déchiffrement » (Bourdieu \& Delsaut 1981), c'està-dire qu'elle suppose une socialisation déjà effective en matière de réception des œuvres chorégraphiques ou du spectacle vivant. Or les étudiant·e-s qui avaient choisi cet atelier optionnel dans l'orientation "Culture et communication » du master en sciences sociales étaient peu familier-ère-s du théâtre et encore moins de la danse. Les faire participer à un processus de production artistique impliquait ainsi un double travail, non seulement pour l'acquisition de techniques du corps, mais également pour l'acquisition d'un savoir minimum sur le champ artistique dans lequel ils et elles allaient évoluer tout au long de ce semestre. Lors du premier cours, seul.e.s trois étudiant·e.s sur vingt avaient une pratique régulière de spectateur ou spectatrice dans les salles de la région, et encore cela ne concernait qu'exclusivement les productions théâtrales. La plupart avait davantage l'habitude de fréquenter les concerts et festivals de musiques actuelles.

Toutefois, rapidement et pour beaucoup s'est constitué un regard de spectateur/spectatrice fondé sur l'expérience pratique de la production esthétique, donc un regard de spectateur-danseur. Lors d'une représentation au théâtre où nous avions l'habitude de répéter, les étudiant.e.s juste sorti.e.s du spectacle auquel nous avions assisté ensemble n'ont pas hésité à émettre des jugements plutôt négatifs sur la pièce qu'ils venaient de voir : " on fait la même chose en répétition », " on reconnaît leur truc » ou bien « franchement si c'est ça des professionnels, je peux dire que je suis danseuse ». La compétence de spectateur est progressivement structurée et permet 
à la plupart des étudiant·e.s de se sentir autorisée.e.s à produire un jugement esthétique informé par le partage d'expériences. L'expérience du travail de création, la coprésence avec des artistes (danseurs et danseuses, chorégraphes, musiciens) a en effet donné à la plupart des étudiant·e.s le sentiment de vivre une expérience sociale peu commune dans le cadre de leur trajectoire académique. Mais comme le souligne une étudiante : «On n'a pas très bien compris ce que la socio foutait là-dedans. Le matin, on revenait sur des notions de sociologie des professions artistiques mais malgré ça, le lien était difficile à faire. C'était nouveau par rapport à la thématique et par rapport à la méthode (ethnographique) que vous proposiez... II y avait d'un côté l'intérêt pour la question artistique et de l'autre côté la non-compréhension de ce qu'était faire de la socio de cette manière-là. »

Le projet, reposant sur l'idée que « l'expérience ethnographique transforme les étudiants en sociologues » (Renahy 2016), s'est heurté à différents écueils. Tout d'abord, le poids de la socialisation scolaire qui isole les savoirs du corps dans une sphère sociocognitive éloignée de l'apprentissage légitime. Décloisonner l'expérience pédagogique en la transférant des salles de cours de l'université aux studios du théâtre a accentué cette distance ressentie par les étudiant·e.s. Ensuite, nous avons progressivement pris conscience du fait que la faible présence de l'enquête de terrain dans leur cursus les obligeait à s'approprier ces nouvelles méthodes d'enquête en même temps que des techniques du corps inconnues. Enfin, à cette absence d'initiation préalable à la méthode ethnographique se superposait un rapport scolastique au savoir sociologique, moins ancré sur le terrain d'enquête que sur des débats théoriques. Les étudiant·e·s ne voyaient pas comment faire le lien entre cette traditionnelle approche scolastique et ce que nous leur proposions, et ce malgré nos digressions, remarques et discussions pendant les moments de pratique chorégraphique et/ou musicale. Notre investissement important, nous faisant faire le double de nos heures (non payées), a été perçu par les étudiants comme une déclinaison de notre vocation artistique et non comme l'expression de notre volonté pédagogique de transmettre, par le biais de l'observation participante, à la fois une méthode d'enquête qui nécessite du temps, et les ressorts de ce qu'était «le terrain ", vocable répété pendant toutes leurs études mais non ancré dans une véritable expérience pratique.

Nous n'avions pas mesuré, au départ, le malentendu entre nos objectifs et la réception du projet par nos étudiant.e.s. Cette expérience que nous voulions indissociablement pédagogique et artistique a en partie échoué dans son ambition d'initiation à la réflexivité sociologique. Elle pose ainsi la question des conditions de possibilité de transmission de la méthode d'enquête ethnographique.

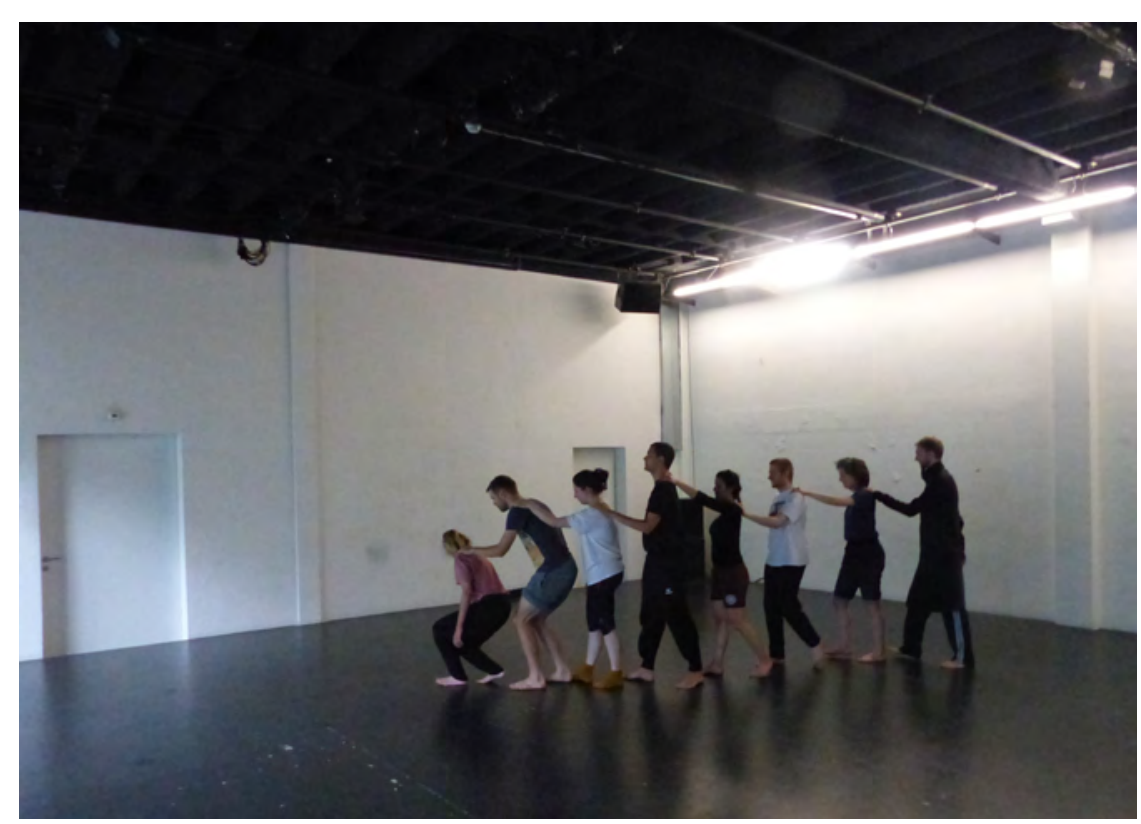

Figure 1. Travail en studio, mars 2016

Les yeux fermés, les étudiant·e.s suivent en « procession » les déplacements de la première de la file. 


\section{Ce que la sociologie fait à l'art : jeux et enjeux institutionnels et esthétiques}

Nous aborderons à présent plus brièvement un autre aspect de cette expérience pédagogique en évoquant la façon dont elle s'est inscrite dans le champ de la danse contemporaine de l'agglomération lausannoise.

\section{Les institutions culturelles et académiques : un jeu gagnant-gagnant?}

Les institutions culturelles que nous avons contactées connaissaient déjà l'un d'entre nous (Sorignet) qui mène une activité d'interprète en France et nouvellement de chorégraphe dans la région. II est également inséré dans les associations culturelles locales de danse contemporaine ainsi qu'enseignant dans une école supérieure de danse à Lausanne. Sa multipositionnalité ainsi que les liens tissés avec certains des directeurs de théâtre qui programment de la danse ont largement aidé à faire en sorte que s'ouvrent, de façon bienveillante, les portes de deux institutions reconnues dans le canton de Vaud. Forts des propositions de partenariat de deux structures prestigieuses du spectacle vivant contemporain à Lausanne, nous avons pu déposer à l'université une demande de Fonds d'innovation pédagogique de 10000 francs suisses (environ 8500 euros), montant essentiellement destiné à couvrir les frais de déplacement et les émoluments des intervenant $\cdot e \cdot s$ invité $\cdot e \cdot s^{2}$. Les responsables du Centre de soutien à l'enseignement auprès de qui nous avons déposé cette demande ont été séduits par notre

2 Comme mentionné en introduction, nous avons activé nos réseaux pour faire venir des artistes de haut niveau (danseurs et danseuses, chorégraphes, musiciens) menant des carrières internationales mais avec qui nous avons des liens d'amitié remontant parfois à vingt ans. Ils et elles ont accepté de participer au projet pour des rémunérations minimales. projet et ont tout de suite soutenu notre atelier de recherche qui constituait à leurs yeux un parfait exemple de « décloisonnement » de l'enseignement supérieur. Nous avons obtenu notre financement rapidement et sans difficulté.

La Suisse jouit d'une prospérité bien supérieure à la quasi-totalité des pays du monde et la relative abondance des ressources qui la caractérise permet en partie d'expliquer les largesses dont nous avons bénéficié en matière d'accès à des structures (studios de danse équipés) et des financements. Mais au-delà d'un contexte matériel particulièrement favorable dont on peut considérer qu'il serait difficile de le retrouver ailleurs (en France notamment), il nous est rapidement apparu que nous entrions avec ce projet dans un jeu d'import-export de capital symbolique entre institutions culturelles et académiques. De fait, les universités suisses tenant le rôle de reproduction des élites sociales dévolu aux « grandes écoles » en France, elles sont des lieux de pouvoir, et l'engagement d'une structure de diffusion culturelle dans un partenariat avec des enseignants-chercheurs sur un projet artistico-pédagogique peut constituer un élément fort de légitimation et de promotion pour les théâtres en question. Réciproquement, une tendance lourde dans le management de la recherche, en Suisse comme ailleurs, n'a de cesse de promouvoir la "créativité ", " l'innovation » et la nécessité de développer des «passerelles arts-sciences » sur lesquelles circuleraient en toute fluidité les membres de la supposée « classe créative » (Florida 2002) censés se stimuler mutuellement. Dès lors, on voit se développer (notamment en Suisse) des " synergies" qui sont autant d'opérations d'import-export de capital symbolique, telles ces résidences d'artistes plasticien-ne-s organisées depuis des années au Centre européen de recherche nucléaire (CERN) de Genève, ou bien ces programmes d'improvisation artistique pour élèves ingénieurs de l'École polytechnique fédérale de Lausanne (EPFL). 


\section{Dénaturaliser la production esthétique}

Dans ce contexte, notre projet a pu encore une fois générer des malentendus. En effet, nous étions bien conscients de l'ambivalence de notre position face à des acteurs institutionnels (directeurs, responsables de la « médiation culturelle » dans les théâtres, ou gestionnaires académiques à l'université) qui attendaient de nous que nous jouions benoîtement le jeu à somme positive dans lequel, par une sorte de magie sociale, « l'art » et "la science » s'inspirent réciproquement. On aura bien compris que notre intention était tout autre, et que l'objectivation des conditions de possibilité de notre atelier (l'intérêt réciproque des institutions à entrer dans ce partenariat) faisait partie des premiers éléments à prendre en compte dans notre analyse réflexive. C'est ainsi que tout au long du semestre nous avons dû jouer finement avec nos hôtes qui attendaient visiblement de nous la production d'un « enchantement » pour " l'art et la culture » auprès de nos étudiant $\cdot e \cdot s$, alors que nous passions le plus clair de notre temps à désenchanter, dénaturaliser, déconstruire les évidences et les catégories des mondes de l'art (le «talent », l'« inspiration », la " créativité », l'« émergence », la « vocation », etc.).

Ainsi lors d'une table ronde consacrée à la fabrique de la programmation, l'un des intervenants, programmateur confirmé, insiste sur la nécessité pour lui d'identifier ce qui est « innovant ». À plusieurs reprises il reprend ce terme, le posant comme la justification première des classements qu'il opère pour faire ses choix. II est alors confronté au questionnement sociologique de certain.e.s de nos étudiant·e.s sur la légitimité qu'il a à désigner ce qui fait « innovation ». Furieux, il nous en gardera une rancune tenace lorsque nous nous adresserons à lui pour négocier une résidence dans son théâtre pour le projet de master 2018.
Plus encore, les orientations esthétiques du spectacle que nous produisions, en retrait, voire en opposition, avec celles valorisées dans l'espace local, nous ont positionnés, malgré nous, dans le champ.

\section{Les enjeux socio-esthétiques}

Traduction en actes du point précédent, nous avons principalement invité des intervenant·e.s qui étaient des " technicien.ne.s du corps » plus que des créateurs conceptuels ${ }^{3}$. II semble exister dans le spectacle vivant contemporain une tendance forte à l'abstraction, à la conceptualisation, tendance qui, à l'instar de ce que la fin du $\mathrm{XX}^{\mathrm{e}}$ siècle a connu dans les arts plastiques avec l'avènement des " curateurs », fait aujourd'hui des programmateurs de salles de spectacle contemporaines des " méta-auteurs » dialoguant avec des metteurs en scène et chorégraphes auteurs, les danseur.se's ayant souvent perdu le rôle qu'ils et elles pouvaient avoir au début du siècle pour devenir des instruments au service de l'auteur. Là encore, notre connaissance professionnelle des mondes du spectacle vivant nous a conduits à analyser la rhétorique de l'enchantement artistique, celle de l'auteur « qui a quelque chose à dire », ou celle de la radicalité conceptuelle au double prisme d'une part de la production sociale des goûts (qu'est-ce qu'un « beau/ bon spectacle » de danse contemporaine et musique à Lausanne en 2016 ?) et d'autre part des savoir-faire de métier des interprètes (qu'est-ce que « danser » ou « jouer de la musique » quand le spectacle est tout entier conceptuel, que le logos a pris le pas sur la praxis ?). C'est aussi en qualité d'interprètes formés pendant des années à la maîtrise d'un corps ou d'un instrument (contrebasse et

3 Nous nous sommes d'ailleurs rendu compte en cours de semestre du fait que la quasi-totalité de nos intervenant·e.s étaient plutôt issu·e.s des classes populaires, ce qui n'est pas anodin dans les mondes de la danse et de la musique contemporaines. 
basse électrique en l'occurrence) que nous avons fait travailler les étudiant·e·s, dans l'idée exposée plus haut qu'il s'agissait moins de transmettre un ethos " artistique » qu'une hexis corporelle et des compétences elles-mêmes susceptibles de commencer à générer un habitus.

Finalement il s'agissait pour nous de faire apparaître le lien entre les conditions de production des œuvres et les œuvres ellesmêmes y compris dans leurs propriétés formelles de manière à montrer l'imbrication des dimensions économique, politique, sociale et esthétique en jeu dans la création contemporaine (Chamboredon 1986). Pour ce faire, les étudiant.e.s devaient devenir spectateurs et spectatrices "éclairée.e.s 》 mais aussi essayer d'objectiver le fonctionnement du champ artistique en enquêtant sur les différents corps de métier qui participent à la production d'une œuvre. Ce que nous n'avions pas mesuré, c'est la réception de notre proposition pédagogique par les prescripteurs de normes esthétiques que sont les programmateurs et dans une moindre mesure les médiateurs culturels (en l'occurrence médiatrices).

Nous avons cherché, d'un commun accord implicite, à produire des formes chorégraphiques qui ne soient ni du théâtre muet ni des happenings conceptuels comme cela peut être le cas dans l'évolution des représentations légitimes de l'art chorégraphique, où la part des " performances " et des propositions dites " conceptuelles " est très importante (surtout en Suisse). Nous avons essayé de faire en sorte que pendant tout le spectacle " il se passe quelque chose " tant au niveau des corps que de l'espace. En cela, nos socialisations professionnelles artistiques ancrées dans l'acquisition de savoir-faire, de techniques du corps autorisant l'expression de la virtuosité (dans la danse comme dans la musique) ont orienté tant notre pédagogie que la fabrique finale du produit esthétique proposé, très éloigné des canons légitimes en vigueur dans les productions diffusées dans les théâtres locaux.

Bien que les deux soirées aient fait salle comble et suscité l'enthousiasme d'un public essentiellement composé des ami-e.s et familles des étudiant.e.s, quasiment aucun'e représentant $\cdot e$ des partenaires, hormis la médiatrice culturelle du théâtre qui nous avait accueilli durant le semestre, ne se sont déplacés, en dépit de leur soutien au projet dans son ensemble. Comment interpréter cette indifférence, alors même que nous avions été accueillis dans leur théâtre ? II faut sans doute ici mettre en perspective la position occupée par l'un de nous deux (Sorignet) : investi dans le champ et identifié comme un « jeune chorégraphe émergent » (sélectionné comme tel dans un programme national en Suisse), il a produit trois projets chorégraphiques soutenus par un petit théâtre local réputé pour sa spécialisation dans la danse contemporaine. II est donc perçu comme un prétendant dans le champ. Or l'esthétique qu'il propose dans ses projets ne semble pas ajustée à celle qui domine localement. En 2017, lors de la renégociation du projet, le jugement négatif porté par quelques acteurs du champ sur la valeur esthétique des chorégraphies de Sorignet aboutit à la nonreconduction du partenariat privilégié que nous avions avec le théâtre qui nous avait accueillis jusqu'alors. II se trouve que ce théâtre venait de renouveler sa direction avec à sa tête un des représentants de l'avant-garde locale (celui-là même qui avait été interrogé par les étudiant·e.s sur sa légitimité à identifier ce qui fait ou non « innovation »). La contestation par ce nouveau directeur du théâtre de notre capacité à proposer aux étudiant·e·s un projet artistique ("vous n'êtes pas vraiment artistes ») et la relégation de nos compétences du côté du savoir académique a éclairé la façon dont nous étions perçus. Attaché à l'autonomie du champ artistique, le nouveau directeur nous refusait la prétention à produire un bien symbolique qui bien qu'étant rattaché à un projet pédagogique 
venait contrecarrer, selon lui, la légitimité des catégories de classement esthétiques, produites par les «professionnel·le·s » de la programmation.

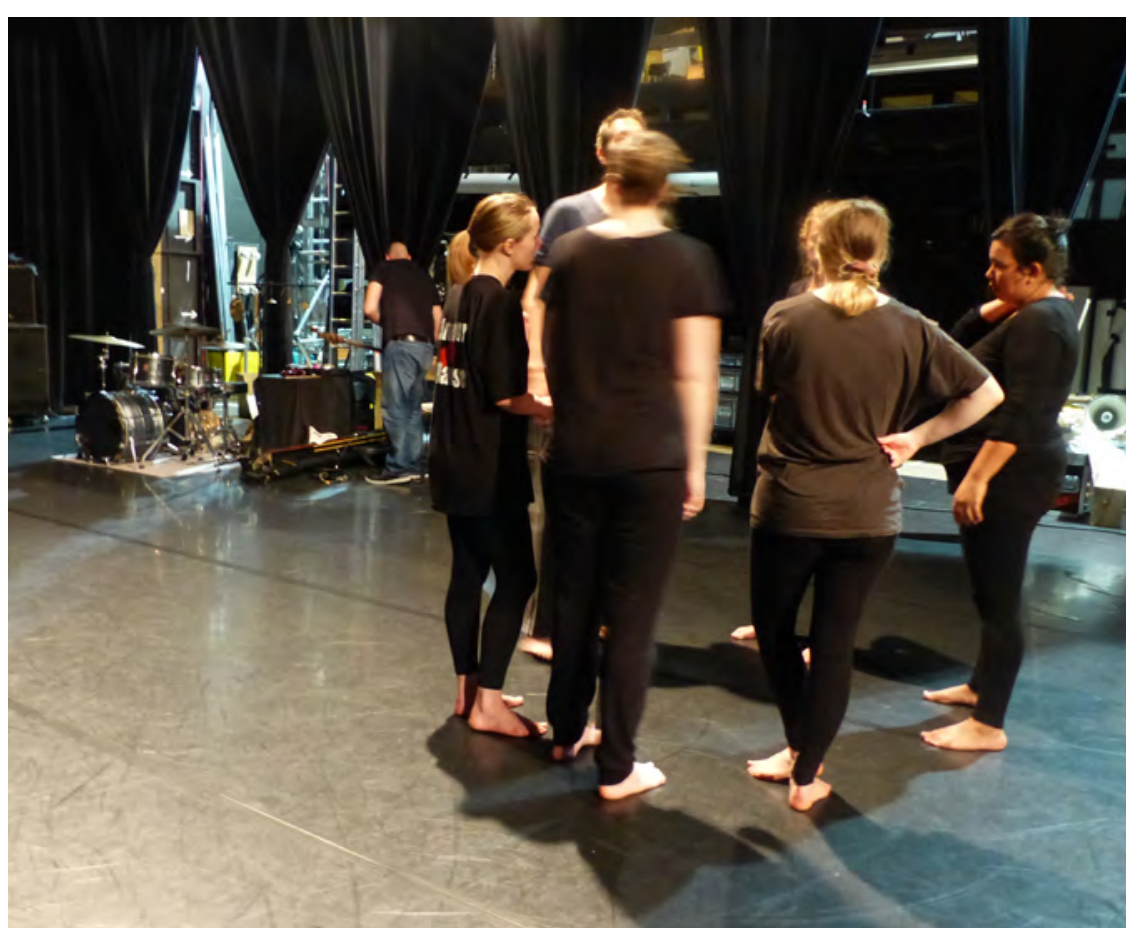

Figure 2. Répétition générale sur le plateau, juin 2016

Lors d'une pause, l'un des enseignants donne des indications à un groupe d'étudiantes.

\section{Conclusion : un dispositif pérenne ?}

L'atelier de recherche en sociologie du travail artistique dans le cadre du master de sciences sociales de l'Université de Lausanne aura lieu tous les deux ans. Notre projet de transmettre une épistémologie et des méthodes d'investigation est intact mais il nous est apparu que nous devions mieux prendre en compte l'écart entre ce que nous pouvions proposer et ce à quoi nos étudiant.e.s étaient formé·e.s. La recherche ethnographique, l'enquête de terrain réflexive, constituent les fondements empiriques des sciences sociales telles que nous les pratiquons, la transmission des savoirs et savoir-faire indispensables à l'exercice de ces méthodes et à l'appréhension d'espaces sociaux tels que les mondes et champs artistiques est toujours notre priorité.

D'autre part, sur le plan institutionnel, il est apparu que les jeux politiques à la tête des équipements culturels et des instances académiques allaient avoir une importance déterminante quant à la possibilité de poursuivre notre projet de manière pérenne. Les attentes des institutions en matière de célébration mutuelle et de profits symboliques n'ont probablement été que partiellement satisfaites, même si l'atelier, notamment grâce aux deux représentations publiques du mois de juin, a acquis une notoriété et une visibilité importantes pour les étudiants (la direction de l'une de nos institutions de tutelle parlera de « vitrine »).

La reconduction du projet pédagogique, si elle est actée pour 2018, se déroule néanmoins dans un environnement doublement problématique. D'une part, une partie du champ artistique apparaît relativement méfiant par rapport à cette initiative : certain·e·s en ont effectivement saisi la dimension critique potentielle tout en restant intéressé.e.s par le savoir sur leur propre structure qu'ils pourraient en retirer. Ainsi en échange d'une courte résidence dans un théâtre lausannois prestigieux, nous avons dû nous engager à faire faire à nos étudiant·e.s une petite enquête sur les publics de la structure. D'autre part, dans l'espace académique, et malgré le soutien de la plupart de nos pairs, une partie de notre hiérarchie semble rester sceptique quant à l'intérêt de ce projet, certes répondant bien aux injonctions à l'« innovation pédagogique » auxquelles 
nous sommes largement exposés, mais contrevenant en même temps aux usages localement dominants tant dans la recherche (l'observation participante étant une méthode méconnue et peu reconnue), que dans l'enseignement (en ce qu'on peut nous soupçonner de « nous amuser "... ce qui semble tout à fait contreindiqué).

Syllabus de l'atelier de recherche (rédigé en janvier 2016) Atelier de recherche Master « Sociologie des professions artistiques "

\section{UNIL - Printemps 2016}

Enseignants :

Marc Perrenoud, Pierre-Emmanuel Sorignet

Emails : Marc.perrenoud@unil.ch, Pierre-emmanuel.sorignet@ unil.ch

\section{Les objectifs de cet enseignement}

Les sciences sociales visent à étudier des faits sociaux, c'est-àdire des faits qui dépassent les individus, qui se rapportent aux différences entre les groupes auxquels ils appartiennent, ou aux relations entre eux.

Mais les sciences sociales ne sont pas un simple ensemble de théories. Faire de la sociologie, de la science politique ou de l'anthropologie, c'est d'abord quelque chose de très pratique. Pour dire quelque chose sur la réalité sociale il faut aller l'observer. L'opportunité de mener cet atelier au théâtre de l'Arsenic nous permet d'ancrer les approches conceptuelles dans les dimensions pratiques et routinières du fonctionnement d'un théâtre. C'est auss la possibilité de partager (un peu) l'un des aspects essentiels du travail artistique, celui de l'activité de production esthétique.

L'objectif de l'atelier est de donner une vision synoptique des métiers du spectacle vivant. Conçu comme un espace d'expérimentation pédagogique, sociologique et artistique, ce cours conduira les étudiant·es à participer à la réalisation d'un projet artistique et scénique. II s'agit pour chacun des participants du séminaire de se mettre en jeu dans les différentes dimensions d'une production artistique.

Encadrés par les deux enseignants qui seront aussi directeurs artistiques, l'un étant danseur et l'autre musicien, enrichis par les interventions d'autres professionnels de la scène (programmateurs, chorégraphes, compositeurs, directeurs de théâtre), les étudiant-e.s occuperont différentes fonctions nécessaires à l'exercice professionnel du spectacle vivant : danser ou jouer de la musique sur scène, mais aussi travailler à la technique (lumière, son), monter un dossier de presse, un plan de communication, travailler sur les aspects logistiques, etc. Les quatorze semaines de cours donneront lieu à un travail intensif, parfois en dehors des horaires académiques et toujours dans des lieux de spectacle, pour aboutir début juin à la présentation d'une performance élaborée collectivement pendant le semestre.

\section{Évaluation et travail demandé}

Le choix de positionner cet enseignement sous un angle expérimental conduit à ajuster la demande faite aux étudiant·e.s.

1. Régularité et engagement

La plus grande régularité sera demandée aux étudiant·es. Le temps imparti étant très court pour prétendre produire un travail collectif de qualité, il est indispensable que toutes les étapes de travail soient acquises dans la même temporalité par chacun.

2. Tenir un journal de terrain

Ce séminaire sera l'occasion de mettre en pratique l'un des principes de l'objectivation en sciences sociales, à savoir la réflexivité. Cette réflexivité sera, en retour, mobilisée dans la recherche artistique. Chaque participante au séminaire tiendra un journal de bord qui sera exploité lors de réflexions communes. C'est donc un travail à la fois personnel et collectif qui sera demandé à tou.te-s. Une dizaine de pages de ce journal de terrain avec des commentaires interprétatifs mobilisant le regard sociologique seront demandées fin mai aux étudiant·e.s. Les étudiant·e-s pourront régulièrement, 
lors des séances, nous demander des précisions quant à l'écriture de ce journal et l'interprétation qu'ils peuvent en faire. Le travail sera à rendre fin mai.

3. Une note de recherche

Une note de recherche concernant l'un des segments des métiers de la scène sera demandé. Les professions artistiques proprement dites (des comédiens aux danseurs en passant par les musiciens) les techniciens du spectacle, mais aussi les intermédiaires du marché que sont les métiers administratifs, en passant par les attachés de presse, les chargés de diffusion, jusqu'aux programmateurs et les journalistes sont susceptibles d'être des objets de recherche. II est requis lors de cette note de recherche au moins deux entretiens et une observation restituée. La possibilité d'avoir accès à ces différents métiers rassemblés au sein de l'Arsenic représente une facilité d'enquête mais implique aussi une présence forte sur les lieux qui ne saurait se limiter au mardi. Une mise en perspective bibliographique sera requise dans cette note de recherche (des bibliographies indicatives vous sont proposées). Cette note de recherche doit être comprise entre 20000 et 30000 signes. Nous encourageons le travail en binôme, voire à trois si l'objet et/ou la méthode choisis le justifient.

4. Les usages du regard sociologique dans un travail artistique L'ambition de cetatelierestdemobiliser le regard sociologiquetantdans la démarche artistique que dans sa dimension plus spécifiquemen académique. Si une partie proprement d'apprentissage des concepts et des travaux de sociologie de la culture sera proposée le matin, c'est tout au long de la journée d'atelier que s'imbriquera le travail sur le plateau et des moments de réflexivité ou de mobilisation de tel ou tel concept sociologique qui permettra de donner une interprétation à des moments de scène autant singuliers que collectifs.

On s'appuiera sur les travaux d'Erving Goffman comme Les Rites d'interaction et La Mise en scène de la vie quotidienne mais aussi sur ceux de Pierre Bourdieu, en particulier La Distinction, ainsi que sur ses écrits autour de la nécessaire réflexivité de l'enquêteur dans son rapport à l'enquête. Ces deux approches seront vues, davantage que complémentaires, comme profondément imbriquées l'une avec l'autre.

Une thématique de création sera dégagée au cours du troisième atelier.

Pierre-Emmanuel Sorigne

Université de Lausanne - LACCUS (Laboratoire capitalisme, culture et sociétés)

$$
\text { Marc Perrenoud }
$$

Université de Lausanne - LACCUS (Laboratoire capitalisme, culture et sociétés)

\section{Bibliographie indicative : sociologie des professions artistiques}

Avril Christelle, Cartier Marie, Serre Delphine (2010). Enquêter sur le travail. Concepts, méthodes, récits. Paris, La Découverte.

BeCKER Howard S. (1985) [1963]. Outsiders. Études de sociologie de la déviance. Paris, Métailié.

BeCKeR Howard S. (1988) [1982]. Les Mondes de l'art. Paris, Flammarion, BOURDIEU Pierre (1977). « La production de la croyance ». Actes de la recherche en sciences sociales, $13: 3-43$.

BOURDIEU Pierre (1977). « Remarques provisoires sur la perception sociale du corps ». Actes de la recherche en sciences sociales, 14

BouRdieu Pierre (1979). La Distinction. Critique sociale du jugement. Paris, Minuit.

BOURDIEU Pierre (1994). «L'économie des biens symboliques ». Raisons pratiques. Paris, Seuil.

CoUlANGEON Philippe (2004). « L'expérience de la précarité dans les professions artistiques. Le cas des musiciens interprètes ». Sociologie de l'art, 3 : 77-110. 
DuBols Vincent (2013. La Culture comme vocation. Paris, Raisons d'agir, « Cours \& Travaux ».

FAURE Sylvia (2003). Apprendre par corps : les modalités d'incorporation des gestes de danse. La Rochelle, Éditions de la Maison des sciences de l'homme.

MengeR Pierre-Menger (1989). « Rationalité et incertitude de la vie d'artiste ». Année sociologique, 39.

Menger Pierre-Menger (1997). La Profession de comédien. Paris, Ministère de la Culture et de la Communication, Département des études et de la prospective.

MAuger Gérard (2006). «Préface ». L'Accès à la vie d'artiste. Sélection et consécration artistiques. Bellecombe en Bauges, Éditions du Croquant, «Champ social ».

Perrenoud Marc (2007). Les Musicos. Enquête sur des musiciens ordinaires. Paris, La Découverte, « Textes à l'appui/enquêtes de terrain ».

Perrenoud Marc (2015). « Économie des biens symboliques et dramaturgie sociale du travail ». In QuiJoux Maxime (dir.), Bourdieu et le travail. Rennes, Presses Universitaires de Rennes (PUR).

SAPIRO Gisèle (2007). « La vocation artistique entre don et don de soi ». Actes de la recherche en sciences sociales, 168.

Schotté Manuel (2012). La Construction du «talent». Sociologie de la domination des coureurs marocains. Paris, Raisons d'agir.

SERRE Delphine (2012). « Le capital culturel dans tous ses états ». Actes de la recherche en sciences sociales, 191-192.
Sinigaglia Jérémy (2012). Artistes, intermittents, précaires en lutte. Retour sur une mobilisation paradoxale. Nancy, Presses Universitaires de Nancy, « Salariat et transformations sociales ».

SORIGNET Pierre-Emmanuel (2011). «Quand la vocation se fait double ». In NAUDIER Delphine \& SIMONET Maud (2011). Des sociologues sans qualités. Paris, La Découverte.

SoRIGNET Pierre-Emmanuel (2012). Danser. Enquête dans les coulisses d'une vocation. Paris, La Découverte, « Textes à l'appui/enquêtes de terrain ».

\section{Manuels d'enquête de terrain à consulter}

Arborio Anne-Marie \& FourniER Pierre (2005). L'Enquête et ses méthodes : l'observation directe. Paris, Armand Colin.

BeAud Stéphane \& Weber Florence (2003). Guide de l'enquête de terrain. Paris, La Découverte.

Becker Howard S. (2002). Les Ficelles du métier. Comment conduire sa recherche en sciences sociales. Paris, La Découverte.

BlANCHEt Alain \& Gotman Anne (1992). L'Enquête et ses méthodes l'entretien. Paris, Nathan.

Combessie Jean-Claude (2007). La Méthode en sociologie. Paris La Découverte, « Repères », $5^{\mathrm{e}}$ éd.

\section{Références bibliographiques}

AvAnza Martina \& LAFERTÉ Gilles (2005). « Dépasser la "construction des identités" ? Identification, image sociale, appartenance ». Genèses, 61(4) : 134-152. 
BECKER Howard S. (1988) [1982]. Les Mondes de l'art. Traduit par Jeanne Bouniort. Paris, Flammarion.

Bourdieu Pierre \& Delsaut Yvette (1981). "Pour une sociologie de la perception ». Actes de la recherche en sciences sociales, $40: 3-9$.

BOURDiEU Pierre (1967). "Système d'enseignement et système de pensée ». Revue internationale des sciences sociales, XIX(3) : 367-388

CHAmboredon Jean-Claude (1986). «Production symbolique et formes sociales. De la sociologie de l'art et de la littérature à la sociologie de la culture ». Revue française de sociologie, 27(3) : 505-529.

Delbos Geneviève \& JoRION Paul (1984). La Transmission des savoirs. Paris, Éditions de la Maison des sciences de l'homme.

DuBoIs Vincent (2013). La Culture comme vocation. Paris, Raisons d'agir.

DuRKHEIM Émile (1969) [1938]. L'Évolution pédagogique en France. Paris, Presses Universitaires de France (PUF).

DuRKHeIM Émile (2005) [1922]. Éducation et sociologie. Paris, Presses Universitaires de France (PUF).

DuRKheIM Émile (2012) [1925]. L'Éducation morale. Paris, Presses Universitaires de France (PUF).

ELIAS Norbert (1973) [1939]. La Civilisation des mœurs. Traduit par Pierre Kamnitzer. Paris, Calmann-Lévy.

FAURE Sylvia (2000). Apprendre par corps. Socio-anthropologie des techniques de danse. Paris, La Dispute.

FAURE Sylvia \& GARCIA Marie-Carmen (2005). Culture hip-hop, jeunes des cités et politiques publiques. Paris, La Dispute.
FIlLoux Jean-Claude (1994). Durkheim et l'éducation. Paris, Presses Universitaires de France (PUF).

FLORIDA Richard (2002). The Rise of the Creative Class. New York, Basic Books.

LAILLIER Joël (2011). " Des familles face à la vocation. Les ressorts de l'investissement des parents des petits rats de l'Opéra ". Sociétés contemporaines, 82 : 59-83.

RENAHY Nicolas (2016). " L'ethnographie comme expérience de conversion à la sociologie : Entretien avec Olivier Schwartz ». Cahiers de recherche sociologique, $61: 191-215$.

SAPIRO Gisèle (2007). "La vocation artistique entre don et don de soi 》. Actes de la recherche en sciences sociales, $168: 4-11$.

SuAUD Charles (1978). La Vocation. Conversion et reconversion des prêtres ruraux. Paris, Minuit.

WACQUANT Loïc (2005). "Carnal Connections : On Embodiment, Apprenticeship, and Membership ». Qualitative Sociology, 28(4) : 445-474. 